\section{Genome Resources for Seven Fungal Isolates That Cause Dollar Spot Disease in Turfgrass, Including Clarireedia jacksonii and C. monteithiana}

\author{
Jo Anne Crouch, ${ }^{1, \dagger}$ Lisa A. Beirn, ${ }^{2}$ Michael J. Boehm, ${ }^{3}$ Ignazio Carbone, ${ }^{4}$ Bruce B. Clarke,${ }^{2}$ James \\ P. Kerns, ${ }^{4}$ Martha Malapi-Wight, ${ }^{1}$ Thomas K. Mitchell, ${ }^{3}$ R. C. Venu, ${ }^{3}$ and Lane P. Tredway ${ }^{4}$ \\ ${ }^{1}$ Mycology and Nematology Genetic Diversity and Biology Laboratory, United States Department of \\ Agriculture-Agricultural Research Service, Beltsville, MD 20705 \\ ${ }^{2}$ Department of Plant Biology, Rutgers University, New Brunswick, NJ 08901 \\ ${ }^{3}$ Department of Plant Pathology, The Ohio State University, Columbus, $\mathrm{OH} 43210$ \\ ${ }^{4}$ Center for Integrated Fungal Research, Department of Entomology and Plant Pathology, North Carolina \\ State University, Raleigh, NC 27695
}

\begin{abstract}
Fungi in the genus Clarireedia are widespread and destructive pathogens of grasses worldwide, and are best known as the causal agents of dollar spot disease in turfgrass. Here, we report genome assemblies of seven Clarireedia isolates, including ex-types of the two most widespread species, Clarireedia jacksonii and $C$. monteithiana. These datasets provide a valuable resource for ongoing studies of the dollar spot pathogens that include population diversity, host-pathogen interactions, marker development, and disease control.
\end{abstract}

Dollar spot is a destructive and widespread fungal disease affecting turfgrasses worldwide (Smiley et al. 2005). Outbreaks of dollar spot disease compromise the health of at least 35 species of warm- and cool-season grasses (Smiley et al. 2005). However, turfgrass species used for golf course fairways and greens incur the greatest economic losses; these include cool-season grasses such as bentgrasses, bluegrasses, and fescues (Agrostis, Poa, and Festuca spp., respectively) and warm-season grasses such as bermudagrass and seashore paspalum (Cynodon dactylon and Paspalum vaginatum, respectively). Symptoms of dollar spot disease are very similar regardless of host, and include leaf lesions that coalesce into distinctive sunken circular patches of turf the approximate size of a silver dollar coin (Smiley et al. 2005).

Dollar spot can be caused by at least five sister species of fungi in the genus Clarireedia (Rutstroemiaceae, Ascomycota) (Hu et al. 2019; Salgado-Salazar et al. 2018), formerly

${ }^{\dagger}$ Corresponding author: J. A. Crouch; joanne.crouch@usda.gov

Current address of L. A. Beirn and L. P. Tredway: Syngenta Crop Protection, Greensboro, NC 27409.

Current address of M. J. Boehm: Department of Plant Pathology, Institute of Agriculture and Natural Resources, University of Nebraska, Lincoln, NE 68588.

Current address of M. Malapi-Wight: United States Department of Agriculture-Animal and Plant Health Inspection Service, Biotechnology Regulatory Services, Riverdale, MD 20737.

Current address of R. C. Venu: RiceTec Inc., Alvin, TX 77512.

Mention of trade names or commercial products in this publication is solely for the purpose of providing specific information and does not imply recommendation or endorsement by the USDA. USDA is an equal opportunity provider and employer.

The author(s) declare no conflict of interest.

Accepted for publication 17 July 2020.

This article is in the public domain and not copyrightable. It may be freely reprinted with customary crediting of the source. The American Phytopathological Society, 2021.
Funding

This work was supported by the United States Department of Agriculture-Agricultural Research Service (USDA-ARS) project 804222000-279-00D, Rutgers Center for Turfgrass Science, North Carolina State Center for Turfgrass Environmental Research and Education, The Ohio State University, and the Ohio Turfgrass Foundation.

\section{Keywords}

Agrostis stolonifera, Cynodon dactylon, dollar spot, Festuca rubra, fungi, Paspalum vaginatum, pathogen diversity, Rutstroemiaceae, Sclerotinia homoeocarpa, turf, turfgrass 
known collectively as Sclerotinia homoeocarpa (Salgado-Salazar et al. 2018). Clarireedia jacksonii and $C$. monteithiana are the most widespread species, affecting cool-season and warm-season grasses, respectively (Salgado-Salazar et al. 2018). Three additional species have been described, each known only from a relatively narrow geographic region or host range: $C$. homoeocarpa, known only from Festuca rubra in the United Kingdom; $C$. bennettii, known from Poaceae hosts in the United Kingdom, United States, and The Netherlands (Salgado-Salazar et al. 2018); and C. paspali, known only from P. vaginatum in China (Hu et al. 2019). Two distinct, single-isolate Clarireedia lineages from $F$. rubra turfgrass in the United Kingdom have also been identified but have not been named (Salgado-Salazar et al. 2018).

The recent taxonomic account of the genus Clarireedia resolves a long unanswered question about the identity of the dollar spot pathogen. At the same time, the recognition that several distinct Clarireedia spp. can infect turfgrasses and cause dollar spot creates a unique opportunity for scientists to dissect the complex cellular underpinnings of disease and host resistance. Disease prediction models, control strategies, population assessments, breeding, diagnostic tools, and other resources are largely based around the concept that just one organism causes dollar spot (Bonos et al. 2006; Green et al. 2016; Rioux et al. 2014; Smith et al. 2018) but some evidence is emerging that phenotypic differences are connected to different Clarireedia spp. (Aynardi et al. 2019).

Here, we report the genome sequences of seven Clarireedia isolates as a resource to enable advanced research of dollar spot disease in turfgrass. Genome sequences from seven isolates of Clarireedia spp. taken from four turfgrass hosts were generated (Table 1). Briefly, three isolates of the globally widespread cool-season grass pathogen $C$. jacksonii were sequenced, including the species ex-type LWC-10 (CBS 138618) (Salgado-Salazar et al. 2018) and the well-studied strain MB-01 (CBS 139392) (Groben et al. 2020; Orshinsky et al. 2012; Ostrander et al. 2014; Putman et al. 2015; Salgado-Salazar et al. 2018; Venu et al. 2011). All C. jacksonii isolates originated from diseased Agrostis stolonifera. Two isolates of the warm-season turf pathogen $C$. monteithiana were sequenced, including the species ex-type RB-19 (CBS 136376). The $C$. monteithiana isolates originated from diseased Cynodon dactylon and $P$. vaginatum turfgrass. Two unnamed Clarireedia spp. isolated from diseased $F$. rubra were also sequenced. Fungal biomass for DNA extraction was harvested by vacuum filtration after 5 days of growth in liquid cultures of yeast extract peptone dextrose (YEPD) media, incubated under constant light and shaking at $150 \mathrm{rpm}$. Genomic DNA $(100 \mu \mathrm{g})$ was extracted from Clarireedia jacksonii MB-01 with the Qiagen Blood \& Cell Culture DNA Kit (Qiagen, Gaithersburg, MD, U.S.A.) following the manufacturer's instructions; for all other isolates, DNA was extracted using the Omni-Pure Genomic DNA Extraction Kit (G-Biosciences, St. Louis, MO, U.S.A.), then purified using the Zymo Genomic DNA Clean \& Concentrator kit (Zymo Research, Irvine, CA, U.S.A.). For RNA extractions, cultures of DRR09 and MB-01 were grown at room temperature under constant light and shaking at $150 \mathrm{rpm}$ for 5 days. Isolate DRR09 was grown in potato dextrose broth (PDB; Thermo Fisher Scientific) and in PDB amended with $2.5 \mathrm{mM}$ ascorbic acid. Isolate MB-01 was grown in YEPD medium (yeast extract at $3 \mathrm{~g} \mathrm{liter}^{-1}$, peptone at $10 \mathrm{~g} \mathrm{liter}^{-1}$, and dextrose at $20 \mathrm{~g} \mathrm{liter}^{-1}$ ). Total RNA was extracted for use for expressed sequence tags (ESTs) using the ZR Fungal/Bacterial RNA MiniPrep kit (Zymo Research). RNA extracted from isolate DRR09 under the two growth conditions was normalized and pooled into a single tube for RNA sequencing.

For the six isolates sequenced using Illumina technology alone, DNA was fragmented using the Covaris M220 ultrasonicator (Covaris, Inc., Woburn, MA, U.S.A.), then used to construct libraries using the TruSeq DNA PCR-free kit (Illumina, Inc., San Diego, CA, U.S.A.). Libraries were sequenced as paired ends using the 600-cycle MiSeq reagent kit on an Illumina MiSeq (Illumina, Inc.). RNA libraries for ESTs were constructed using the TruSeq RNA LT preparation kit (Illumina, Inc.). Libraries were quantified as previously described (Malapi-Wight et al. 2015) and sequenced in two independent runs on the Illumina MiSeq platform to generate $1 \times 50$ and $2 \times 150$ reads. Post-run read processing was performed using CLC Genomics Workbench v. 7.5.1 (CLC Bio, Germantown, MD, U.S.A.). After adapter removal, bases with low Phred quality scores (limit 0.05) and strings of ambiguous bases $>2$ were trimmed, and reads $<15$ bp were removed from the dataset. Between 9.9 and 28.8 million reads were retained after quality filtering. Assembly of Illumina reads was performed in CLC Genomics ( $k m e r=61$, bubble size of 50 ), with scaffolds $\geq 500$ bp retained for these six assemblies generated entirely from Illumina reads. The CLC Microbial Genome Finishing Module was used to join scaffolds based on paired-end data and BLAST alignments of scaffold ends (e-value 10-5, minimum match size 50 bp; Qiagen). 
Table 1. Summary of genome assemblies from seven isolates of Clarireedia $^{a}$

\begin{tabular}{|c|c|c|c|c|c|c|c|}
\hline \multirow[b]{2}{*}{ Variables } & \multicolumn{2}{|c|}{ Clarireedia sp. } & \multicolumn{2}{|c|}{ Clarireedia monteithiana } & \multicolumn{3}{|c|}{ C. jacksonii } \\
\hline & CPB17 & SE16F-4 & DRR09 & RB-19 ${ }^{b}$ & LWC-10 ${ }^{b}$ & MB-01 & SH44 \\
\hline $\begin{array}{l}\text { CBS accession } \\
\text { Host }\end{array}$ & $\begin{array}{l}\text { CBS } 136371 \\
\text { Festuca rubra }\end{array}$ & $\begin{array}{l}\text { CBS } 136372 \\
\text { F. rubra }\end{array}$ & $\begin{array}{c}\text { CBS } 136373 \\
\text { Paspalum } \\
\text { vaginatum }\end{array}$ & $\begin{array}{c}\text { CBS } 136376 \\
\begin{array}{c}\text { Cynodon } \\
\text { dactylon }\end{array}\end{array}$ & $\begin{array}{c}\text { CBS } 138618 \\
\text { Agrostis stolonifera }\end{array}$ & $\begin{array}{l}\text { CBS } 139392 \\
\text { A. stolonifera }\end{array}$ & $\begin{array}{l}\text { CBS } 138617 \\
\text { A. stolonifera }\end{array}$ \\
\hline $\begin{array}{l}\text { Geographic } \\
\text { origin }\end{array}$ & $\begin{array}{l}\text { United } \\
\text { Kingdom }\end{array}$ & $\begin{array}{l}\text { United } \\
\text { Kingdom }\end{array}$ & $\begin{array}{l}\text { Dominican } \\
\text { Republic }\end{array}$ & $\begin{array}{l}\text { Mississippi, } \\
\text { United States }\end{array}$ & $\begin{array}{l}\text { North Carolina, } \\
\text { United States }\end{array}$ & $\begin{array}{c}\text { Ohio, United } \\
\text { States }\end{array}$ & Canada \\
\hline Year collected & 2008 & 2008 & 2008 & 2008 & 2003 & 2001 & 2000 \\
\hline $\begin{array}{l}\text { Assembly size } \\
\text { (Mb) }\end{array}$ & 36.4 & 36.0 & 48.7 & 36.1 & 37.2 & 39.5 & 35.9 \\
\hline $\begin{array}{l}\text { Scaffolds } \geq 500 \\
\text { bp }(n)\end{array}$ & 3,873 & 4,789 & 15,133 & 3,610 & 3,606 & 1,637 & 4,289 \\
\hline $\begin{array}{l}\text { Scaffolds } \geq 1 \\
\mathrm{~kb}(n)\end{array}$ & 2,975 & 3,725 & 9,341 & 2,955 & 2,820 & 1,637 & 3,468 \\
\hline $\begin{array}{l}\text { Average } \\
\text { coverage }\end{array}$ & 41 & 38 & 109 & 33 & 58 & 70 & 41 \\
\hline Scaffold $\mathrm{N}_{50}$ & 20,651 & 15,633 & 8,758 & 20,763 & 25,161 & 168,655 & 17,322 \\
\hline $\begin{array}{l}\text { Maximum } \\
\text { scaffold } \\
\text { length }\end{array}$ & 119,633 & 100,512 & 78,171 & 116,952 & 122,690 & 727,903 & 110,593 \\
\hline $\begin{array}{l}\text { Average } \\
\text { scaffold } \\
\text { length }\end{array}$ & 9,392 & 7,518 & 3,219 & 10,023 & 10,328 & 24,110 & 8,367 \\
\hline Illumina reads $(n)$ & $10,448,372$ & $9,897,838$ & $28,807,323$ & $8,031,180$ & $15,204,474$ & - & $10,256,106$ \\
\hline GC\% & 44.6 & 45.0 & 44.8 & 44.7 & 41.6 & 39.7 & 45.1 \\
\hline Gene models & 11,134 & 11,035 & 16,244 & 10,821 & 11,089 & 10,979 & 10,975 \\
\hline $\begin{array}{l}\text { BUSCO, } \\
\quad \text { eukaryote (\%) }\end{array}$ & 96 & 95 & 94 & 96 & 97 & 97 & 96 \\
\hline $\begin{array}{l}\text { BusCO, fungus } \\
(\%)^{\mathrm{e}}\end{array}$ & 97 & 98 & 97 & 98 & 98 & 98 & 98 \\
\hline
\end{tabular}

$(\%)^{\mathrm{e}}$

GenBank LLKJ00000000 LLKF00000000 LLKI00000000 LLKG00000000

LLKH00000000

LLKD00000000 LLKE00000000

a Clarireedia jacksonii MB-01 was sequenced using a combination of Sanger/lllumina technologies; all other isolates were sequenced using Illumina technology. All genome data are based on scaffolds $\geq 500 \mathrm{bp}$, except for $C$. jacksonii MB-01, which is limited to scaffolds $\geq 1 \mathrm{~kb}$.

b Ex-type for the species.

${ }^{c}$ All isolates are deposited in the CBS-KNAW Collection, Westerdijk Fungal Biodiversity Institute, The Netherlands.

d BUSCO analysis based on a set of 438 eukaryote orthologs.

e BUSCO analysis based on a set of 1,438 fungal orthologs.

Paired-end (lllumina) and mate-paired (Sanger;12 kb) DNA libraries of $C$. jacksonii isolate MB01 were constructed, sequenced, and assembled by the J. Craig Venter Institute (Rockville, MD, U.S.A.). Multiple assemblies using multiple assemblers and parameter variations were performed to obtain the highest-quality assembly. This included one CLC assembly using only Illumina pairedend reads, three CLC assemblies using both Illumina reads and Sanger-sequenced mate pairs with varying parameters of mate-pair distance and treatment of nonspecific matches, and one Celera Assembler (CA) assembly using the raw, untrimmed lllumina reads combined with Sanger mate-pair information. At the contig level, both CA and CLC performed similarly, with CA yielding an $\mathrm{N}_{50}$ of about $27 \mathrm{~kb}$ while CLC yielded an $\mathrm{N}_{50}$ between 27 and $43 \mathrm{~kb}$ for the different runs (scaffolds $\geq 1 \mathrm{~kb}$ ). Overall, CA produced the best MB-01 assembly, comprising 1,637 scaffolds, a scaffold $\mathrm{N}_{50}$ of about $168 \mathrm{~kb}$, and an average genome coverage of 70x.

Relative to other members of the Rutstroemiaceae and Sclerotiniaceae families, the seven Clarireedia genome assemblies fell within the overall size range of 38.3 to $51.9 \mathrm{Mb}$ (Amselem et al. 2011; Blanco-Ulate et al. 2013; Green et al. 2016; Mardanov et al. 2014; Nest et al. 2014). With the exception of $C$. monteithiana DRR09, genome assembly sizes ranged from 35.9 to $39.5 \mathrm{Mb}$. In contrast, the $C$. monteithiana DRR09 assembly was $48.7 \mathrm{Mb}$ and highly fragmented $(9,341$ scaffolds $>1 \mathrm{~kb})$. The difference in size observed between DRR09 and the other isolates is likely due to the completeness of the assemblies; scaffold accumulation plots generated in QUAST (Gurevich et al. 2013) showed that a plateau was reached for all isolates except DRR09. This is despite the fact that DRR09 was sequenced at a higher depth of coverage than the other six isolates (109x versus $33 \times$ to $70 x$ ). Gene models were predicted using AUGUSTUS v3.0.2 (Stanke and Morgenstern 2005) with S. sclerotiorum gene models (Amselem et al. 2011) and using C. jacksonii MB-01 and C. monteithiana 
DRR09 RNA sequences generated from this study (52,893 and 83,517 ESTs, respectively) and previous work (29,542 ESTs) (Orshinsky et al. 2012). With the exception of DRR09, the number of predicted gene models ranged from 10,821 to 11,134 .

The genome assemblies described herein are contained within a relatively large number of scaffolds (1,637 to 3,725 scaffolds for all isolates except DRR09). However, comparisons with a hybrid 231-scaffold genome assembly of $C$. jacksonii (formerly known as S. homoeocarpa) isolate HRS10 (Illumina/PacBio hybrid) (Green et al. 2016) shows that, although the scaffold numbers are larger, these new genome resources provide a largely complete representation of the overall gene space. BUSCO (Simão et al. 2015) assessment of conserved single-copy orthologs identified 97 to $98 \%$ of the fungal orthologs and 94 to $97 \%$ of the eukaryotic orthologs from the new assemblies. In contrast, the C. jacksonii HRS10 assembly contained 98 and $100 \%$ of the BUSCO eukaryote and fungal ortholog sets, respectively. Similarly, the number of gene models predicted from the new genome resources are comparable with the 11,285 gene models we predicted from the $C$. jacksonii HRS10 assembly using the same AUGUSTUS pipeline described above.

The genome assemblies described in this article were deposited in the NCBI GenBank under the accessions listed in Table 1. All datasets described herein are permanently curated and publicly available online by the U.S. National Agricultural Library AgData Commons at https://data.nal.usda.gov/dataset/data-genome-resources-seven-fungal-isolates-cause-turfgrass-dollar-spotdisease-including-clarireedia-jacksonii-and-c-monteithiana. All Clarireedia isolates were deposited in the CBS-KNAW Collection, Westerdijk Fungal Biodiversity Institute, The Netherlands.

\section{Literature Cited}

Amselem, J., Cuomo, C. A., van Kan, J. A. L., Viaud, M., Benito, E. P., Couloux, A., Coutinho, P. M., de Vries, R. P., Dyer, P. S., Fillinger, S., Fournier, E., Gout, L., Hahn, M., Kohn, L., Lapalu, N., Plummer, K. M., Pradier, J. M., Quévillon, E., Sharon, A., Simon, A., ten Have, A., Tudzynski, B., Tudzynski, P., Wincker, P., Andrew, M., Anthouard, V., Beever, R. E., Beffa, R., Benoit, I., Bouzid, O., Brault, B., Chen, Z., Choquer, M., Collémare, J., Cotton, P., Danchin, E. G., Da Silva, C., Gautier, A., Giraud, C., Gonzlez, C., Grossetet, S., Güldener, U., Henrissat, B., Howlett, B. J., Kodira, C., Kretschmer, M., Lappartient, A., Leroch, M., Levis, C., Mauceli, E., Nevéglise, C., Oeser, B., Pearson, M., Poulain, J., Poussereau, N., Quesneville, H., Rascle, C., Schumacher, J., Séguren, B., Sexton, A., Silva, E., Sirven, C., Soanes, D. M., Talbot, N. J., Templeton, M., Yandava, C., Yarden, O., Zeng, Q., Rollins, J. A., Lebrun, M. H., and Dickman, M. 2011. Genomic analysis of the necrotrophic fungal pathogens Sclerotinia sclerotiorum and Botrytis cinerea. PLoS Genet. 7:e1002230.

Aynardi, B. A., Jiménez-Gasco, M. M., and Uddin, W. 2019. Effects of isolates of Clarireedia jacksonii and Clarireedia monteithiana on severity of dollar spot in turfgrasses by host type. Eur. J. Plant Pathol. 155:817-829.

Blanco-Ulate, B., Allen, G., Powell, A. L. T., and Cantu, D. 2013. Draft genome sequence of Botrytis cinerea BcDW1, inoculum for noble rot of grape berries. Genome Announce. 1:e00252-13.

Bonos, S. A., Clarke, B. B., and Meyer, W. A. 2006. Breeding for disease resistance in the major cool-season turfgrasses. Annu. Rev. Phytopathol. 44:213-234.

Green, R., Sang, H., Chang, T., Allan-Perkins, E., Petit, E., and Jung, G. 2016. Draft genome sequences of the turfgrass pathogen Sclerotinia homoeocarpa. Genome Announce. 4:e01715-15.

Groben, G., Clarke, B. B., Murphy, J., Koch, P., Crouch, J. A., Lee, S., and Zhang, N. 2020. Real-time PCR detection of Clarireedia spp., the causal agents of dollar spot in turfgrasses. Plant Dis. 104:3118-3123.

Gurevich, A., Saveliev, V., Vyahhi, N., and Tesler, G. 2013. QUAST: Quality assessment tool for genome assemblies. Bioinformatics 29:1072-1075.

Hu, J., Zhou, Y., Geng, J., Dai, Y., Ren, H., and Lamour, K. 2019. A new dollar spot disease of turfgrass caused by Clarireedia paspali. Mycol. Prog. 18:1423-1435.

Malapi-Wight, M., Salgado-Salazar, C., Demers, J. E., Veltri, D., and Crouch, J. A. 2015. Draft genome sequence of Dactylonectria macrodidyma, a plant pathogenic fungus in the Nectriaceae. Genome Announce. 3:e00278-15.

Mardanov, A. V., Beletsky, A. V., Kadnikov, V. V., Ignatov, A. N., and Ravin, N. V. 2014. Draft genome sequence of Sclerotinia borealis, a psychrophilic plant pathogenic fungus. Genome Announce. 2:e01175-13.
Nest, M. A., Beirn, L. A., Crouch, J. A., Demers, J. E., de Beer, Z. W., Vos, D. V., Gordon, T. R., Moncalvo, J.-M., Naidoo, K., Sanchez-Ramirez, S., Roodt, D., Santana, Q. C., Slinski, S. L., Stata, M., Taerum, S. J., Wilken, P. M., Wilson, A. M., Wingfield, M. J., and Wingfield, B. D. 2014. Draft genomes of Amanita jacksonii, Ceratocystis albifundus, Fusarium circinatum, Huntiella omanensis, Leptographium procerum, Rutstroemia sydowiana and Sclerotinia echinophila. IMA Fungus 5:473-486.

Orshinsky, A. M., Hu, J., Opiyo, S. O., Reddyvari-Channarayappa, V., Mitchell, T. K., and Boehm, M. J. 2012. RNA-Seq analysis of the Sclerotinia homoeocarpacreeping bentgrass pathosystem. PLoS One 7:e41150.

Ostrander, J. C., Todd, R. B., and Kennelly, M. M. 2014. Resistance of Kansas Sclerotinia homoeocarpa isolates to thiophanate-methyl and determination of associated $\beta$-tubulin mutation. Plant Health Prog. 15:80-84.

Putman, A. I., Tredway, L. P., and Carbone, I. 2015. Characterization and distribution of mating-type genes of the turfgrass pathogen Sclerotinia homoeocarpa on a global scale. Fungal Genet. Biol. 81:25-40.

Rioux, R. A., Shultz, J., Garcia, M., Willis, D. K., Casler, M., Bonos, S., Smith, D., and Kerns, J. P. 2014. Sclerotinia homoeocarpa overwinters in turfgrass and is present in commercial seed. PLoS One 9:e110897.

Salgado-Salazar, C., Beirn, L. A., Ismaiel, A., Boehm, M. J., Carbone, I., Putman, A. I., Tredway, L. P., Clarke, B. B., and Crouch, J. A. 2018. Clarireedia: A new fungal genus comprising four pathogenic species responsible for dollar spot disease of turfgrass. Fungal Biol. 122:761-773.

Simão, F. A., Waterhouse, R. M., loannidis, P., Kriventseva, E. V., and Zdobnov, E. M. 2015. BUSCO: Assessing genome assembly and annotation completeness with single-copy orthologs. Bioinformatics 31:3210-3212.

Smiley, R. W., Dernoeden, P. H., and Clarke, B. B. 2005. Compendium of Turfgrass Diseases, 3rd ed. American Phytopathological Society, St. Paul, MN, U.S.A.

Smith, D. L., Kerns, J. P., Walker, N. R., Payne, A. F., Horvath, B., Inguiagiato, J. C., Kaminski, J. E., Tomaso-Peterson, M., and Koch, P. L. 2018. Development and validation of a weather-based warning system to advise fungicide applications to control dollar spot on turfgrass. PLoS One 13:e0194216.

Stanke, M., and Morgenstern, B. 2005. AUGUSTUS: A web server for gene prediction in eukaryotes that allows user-defined constraints. Nucleic Acids Res. 33: W465-W467.

Venu, R. C., Zhang, Y., Weaver, B., Carswell, P., Mitchell, T. K., Meyers, B. C., Boehm, M. J., and Wang, G.-L. 2011. Large scale identification of genes involved in plant fungal interactions using Illumina's sequencing-by-synthesis technology. Methods Mol. Biol. 722:167-178. 